\title{
Population fluctuation of some harmful wild birds in different habitats at Assiut governorate, Egypt
}

\author{
Mosallm M. A. S. ${ }^{1 *}$, Omar M. M. A. ${ }^{1}$, El-Danasory M. A. M. $^{2}$, El-Sharkawy R. M. I. ${ }^{3}$ \\ ${ }^{I}$ Department of Agricultural Zoology and Nematology, Faculty of Agriculture, Al-Azhar University, Assiut, Egypt \\ ${ }^{2}$ Department of Agricultural Zoology and Nematology, Faculty of Agriculture, Al-Azhar University, Cairo, Egypt \\ ${ }^{3}$ Agricultural Botany Department, Faculty of Agriculture, Al-Azhar University, Assiut, Egypt
}

\begin{abstract}
A field experiment was carried out in order to study the population fluctuations of harmful wild birds at four different habitats, i.e., Nearby animal's farm, field crops, honeybee apiary and trees. This study was carried out in planted areas at farm of Faculty of Agriculture, Al-Azhar University, Assiut, Egypt during December 2018 till November 2020. The study proved that there are four species of wild birds it is present throughout the year in the study area. The four species were house sparrow, Passer domusticus niloticus, hooded crow, Corvus corone sardonius, palm dove, Streptopilia senegalensis egyptica and rock dove, Columba livia schimpari. The study showed that the highest population of house sparrow was recorded in fields nearby trees, followed by fields nearby honeybee apiary and fields nearby field crops. Then the lowest population was in fields nearby animal's farm. Also, the population of hooded crow was highest in fields nearby animal's farm and trees, followed by fields nearby honeybee apiary. Then the lowest numbers were recorded in fields nearby field crops. As for the palm dove the highest population were in fields nearby trees, followed by fields nearby honeybee apiary, followed by fields nearby animal's farm. And the lowest population recorded in fields nearby field crops. Finally, the rock dove bird recorded the highest population in fields nearby animal's farm, followed by fields nearby honeybee apiary and fields nearby trees. As for the lowest population of rock dove were in fields nearby field crops.
\end{abstract}

Keywords: population, fluctuation, house sparrow, hooded crow, nearby trees. 


\section{Introduction}

The habitat is important for living organisms in terms of food and shelter that it provides. The distribution of animals varies according to the difference in the habitat and the type of food in this habitat. In Egypt, there are some birds that live in tropical forests, others live around the coasts and some live around fresh lakes, rivers and swamps. The house sparrow, Passer domesticus niloticus (L.) is one of the most important bird residents in Egypt. It is one of the most important agriculture pests in the cultivated areas, such as wheat, barley, rice, broad bean and grapes (Attia, 2013; Hassan, 2018; Khattab et al., 2001; Metwally et al., 2009; Omar 2010). Also, the hooded crow bird in terms of importance in Egypt. It is one of the most important agricultural pests in the cultivated areas, such as maize, peanuts, sunflower, (Bonnah, 2007; Khattab et al., 2002; Metwally et al., 1995). Omar (2010) revealed that house sparrow, palm dove and hooded crow were the most prevalent noxious bird species attacked field crops at ripening stages. The present work was done in the fields of the different habitats at farm of Agriculture Faculty, Al-Azhar University, Assiut, Egypt in order study the population fluctuation of the most important species during December 2018 till November 2020.

\section{Materials and methods}

The harmful bird species were surveyed in four different habitats (animal's farm, honeybee apiary, field crops and trees) during December 2018 till November 2020 at planted areas at farms of Faculty of Agriculture, Al-Azhar University, Assiut, Egypt. In these trails, area size is two feddans (feddan $=4200 \mathrm{~m}^{2}=0.420$ hectares $=1.037$ acres) inside the chosen cultivated Location. Number of the different bird species was counted in each habitat by using the method of Redinger and Libay (1979) as a plot equivalent two feddans from the determined cultivated area in each location. The identification and counts of bird species were achieved by using field glass (binoculars) from rising position, which gave clear sighted vision of the plots. This work has been accomplished twice daily, the first at sunrise and second at sunset during one hour for four days monthly. Population fluctuation of the house sparrow, $P$. domusticus niloticus (L.), hooded crow, C. corone sardonius (L.), palm dove, $S$. senegalensis egyptica (L.) and rock dove, Columba livia schimpari (L.). was studied monthly during daytime at four different habitats nearby (animal's farm, honeybee apiary, field crops and trees) in planted areas during December 2018 till November 2020 two successive years.

\subsection{Statistical analysis}

Data obtained were statistically analyzed using a randomized complete block design. Means were compared according to Duncan's Multiple Range test, at 0.05 level of probability. 


\section{Results and Discussion}

\subsection{Population fluctuation of some} harmful wild birds

Four harmful wild bird species were surveyed in four different habitats, i.e., nearby animal's farm, field crops, honeybee apiary and trees. This study was carried out in planted areas at farm of Faculty of Agriculture, Al-Azhar University, Assiut, Egypt during December 2018 till November 2020.

\subsubsection{Population fluctuation of house sparrow bird Passer domesticus niloticus}

Data in Table (1) indicated that the highest population were in fields nearby trees with means (66.75 and 68.42 individuals) during December 2018 till November 2020 , and that recorded in fields nearby honeybee apiary with means (48.67 and 45.29 individuals), followed by the population in fields nearby field crops with means (22.08 and 21.42 individuals). Then the lowest numbers were recorded in fields nearby animals' farm with means (18.25 and 19.83 individuals) during December 2018 till November 2020. The statistical analysis data for means indicated that there were highly significant differences between the fields nearby trees and fields nearby field crops and fields nearby animal's farm. Also, Data in the same Table showed that the level of abundance of house sparrow birds that recorded in December 2018 compared with 2019 with means (27.63 and 24.75 individuals). In the next months, January and February 2019 and 2020, there was no high significant change in the level of abundance of house sparrow birds that recorded with means (19.50 and 20.50 individuals) and (29.13 and 32.88 individuals). The population began to increase during the month of March 2019 and 2020 with mean (44.28 and 48.13 individuals). Through the month of April 2019 and 2020 the population had reached the highest level with means (72.38 and 68.13 individuals). After reaching the peak in the population of house sparrow bird, it began to decrease gradually during the following three months (May, June and July) 2019 and 2020 with means, (45.38 and 44.00 individuals), (33.25 and 29.00 individuals) and (23.00 and 26.50 individuals). The population returned to increase again with means (41.13 and 32.25 individuals) and (61.75 and 59.25 individuals) in August and September 2019 and 2020. Also, the population returned to decrease gradually during two months (October and November) for 2019 and 2020 with means, (45.25 and 46.88 individuals) and (32.13 and 32.13 individuals). The results agree with Omar (2018) who reported that the highest numbers were (62.71 and 64.88 individuals) in trees, followed by buildings. While the lowest numbers were (18.38 and 16.83 individuals) in fields at Assiut governorate, Egypt. 
Table (1): Population fluctuation and monthly distribution of house sparrow Passer domesticus niloticus: at different habitats in Assiut governorate, Egypt during December 2018 till November 2020.

\begin{tabular}{|c|c|c|c|c|c|c|c|c|c|c|c|c|c|}
\hline \multirow{3}{*}{ Months } & \multicolumn{12}{|c|}{ Different habitats } & \multirow{3}{*}{ Mean } \\
\hline & \multicolumn{3}{|c|}{ Animal's farm } & \multicolumn{3}{|c|}{ Field crops } & \multicolumn{3}{|c|}{ Honeybee apiary } & \multicolumn{3}{|c|}{ Trees } & \\
\hline & S. R. & S. S. & Av. & S. R. & S. S. & Av. & S. R. & S. S. & Av. & S. R. & S. S. & Av. & \\
\hline December 2018 & 16 & 10 & 13.00 & 8 & 6 & 7.00 & 30 & 43 & 36.50 & 47 & 61 & 54.00 & $27.63 d$ \\
\hline January 2019 & 17 & 12 & 14.50 & 17 & 16 & 16.50 & 22 & 17 & 19.50 & 30 & 25 & 27.50 & $19.50 \mathrm{~d}$ \\
\hline February 2019 & 23 & 29 & 26.00 & 18 & 16 & 17.00 & 39 & 34 & 36.50 & 34 & 40 & 37.00 & $29.13 \mathrm{~d}$ \\
\hline March 2019 & 47 & 30 & 38.50 & 22 & 18 & 20.00 & 51 & 48 & 49.50 & 72 & 66 & 69.00 & $44.25 \mathrm{bcd}$ \\
\hline April 2019 & 31 & 32 & 31.50 & 41 & 45 & 43.00 & 99 & 102 & 100.50 & 107 & 137 & 114.50 & $72.38 \mathrm{a}$ \\
\hline May 2019 & 29 & 18 & 23.50 & 30 & 32 & 31.00 & 56 & 33 & 44.50 & 91 & 74 & 82.50 & $45.38 \mathrm{bcd}$ \\
\hline June 2019 & 15 & 21 & 18.00 & 21 & 15 & 18.00 & 21 & 35 & 28.00 & 65 & 77 & 69.00 & $33.25 \mathrm{~cd}$ \\
\hline July 2019 & 9 & 7 & 8.00 & 6 & 6 & 6.00 & 38 & 27 & 32.50 & 49 & 42 & 45.50 & $23.00 \mathrm{~d}$ \\
\hline August 2019 & 22 & 20 & 21.00 & 16 & 24 & 20.00 & 68 & 74 & 71.00 & 46 & 59 & 52.50 & $41.13 \mathrm{bcd}$ \\
\hline September 2019 & 27 & 25 & 26.00 & 42 & 35 & 38.50 & 82 & 79 & 80.50 & 110 & 94 & 102.00 & $61.75 \mathrm{ab}$ \\
\hline October 2019 & 11 & 18 & 14.50 & 36 & 28 & 32.00 & 40 & 51 & 45.50 & 88 & 90 & 89.00 & $45.25 \mathrm{bcd}$ \\
\hline November 2019 & 21 & 8 & 14.50 & 20 & 12 & 16.00 & 33 & 46 & 39.50 & 55 & 62 & 58.50 & $32.13 \mathrm{~cd}$ \\
\hline Mean & 22.33 & 19.17 & 20.75 & 23.08 & 21.08 & \begin{tabular}{|l|}
$22.08 \mathrm{c}$ \\
\end{tabular} & 48.25 & 49.08 & $48.67 \mathrm{~b}$ & 66.17 & 68.92 & $67.54 \mathrm{a}$ & $39.76 \mathrm{a}$ \\
\hline December 2019 & 10 & 9 & 9.50 & 12 & 16 & 14.00 & 36 & 31 & 33.50 & 36 & 48 & 42.00 & $24.75 \mathrm{~d}$ \\
\hline January 2020 & 11 & 13 & 12.00 & 12 & 10 & 11.00 & 27 & 23 & 25.00 & 40 & 32 & 36.00 & $20.50 \mathrm{~d}$ \\
\hline February 2020 & 30 & 17 & 23.50 & 18 & 25 & 21.50 & 37 & 39 & 38.00 & 50 & 47 & 48.50 & $32.88 \mathrm{~cd}$ \\
\hline March 2020 & 26 & 34 & 30.00 & 21 & 27 & 24.00 & 62 & 50 & 56.00 & 79 & 86 & 82.50 & $48.12 \mathrm{abcd}$ \\
\hline April 2020 & 20 & 32 & 26.00 & 38 & 39 & 38.50 & 83 & 96 & 89.50 & 113 & 124 & 118.50 & $68.12 \mathrm{ab}$ \\
\hline May 2020 & 17 & 25 & 21.00 & 32 & 24 & 28.00 & 41 & 45 & 43.00 & 80 & 88 & 84.00 & $44.00 \mathrm{bcd}$ \\
\hline June 2020 & 13 & 18 & 15.50 & 15 & 13 & 14.00 & 22 & 24 & 23.00 & 71 & 56 & 63.50 & $29.00 \mathrm{~d}$ \\
\hline July 2020 & 12 & 15 & 13.50 & 10 & 11 & 10.50 & 35 & 23 & 29.00 & 52 & 54 & 53.00 & $26.50 \mathrm{~d}$ \\
\hline August 2020 & 18 & 22 & 20.00 & 18 & 12 & 15.00 & 46 & 37 & 41.50 & 44 & 61 & 52.50 & $32.25 \mathrm{~cd}$ \\
\hline September 2020 & 24 & 30 & 27.00 & 36 & 43 & 39.50 & 75 & 60 & 67.50 & 97 & 109 & 103.00 & $59.25 \mathrm{abc}$ \\
\hline October 2020 & 23 & 22 & 22.50 & 24 & 22 & 23.00 & 55 & 63 & 59.00 & 76 & 90 & 83.00 & $46.88 \mathrm{bcd}$ \\
\hline November 2020 & 16 & 19 & 17.50 & 17 & 19 & 18.00 & 35 & 42 & 38.50 & 56 & 53 & 54.50 & $32.13 \mathrm{~cd}$ \\
\hline Mean & 18.33 & 21.33 & $19.83 \mathrm{c}$ & 21.08 & 21.75 & $21.42 \mathrm{c}$ & 46.12 & 44.42 & $45.29 \mathrm{~b}$ & 66.17 & 70.67 & $68.42 \mathrm{a}$ & $38.74 \mathrm{a}$ \\
\hline
\end{tabular}

S.R. = sun-rise, S.S. = sun-set, Av. = Average number, *Means have the same litters are not significantly differed by using Duncan's analysis.

\subsubsection{Population fluctuation of hooded crow Corvus corone sardonius}

Data in Table (2) shows that, there was no significant difference in the level of abundance of hooded crow in three habitats, (animal's farm, honeybee apiary and trees) during December 2018 till November 2020 where the means were (3.71 and 3.21 individuals) nearby animal's farm, (3.58 and 3.21 individuals) nearby honeybee apiary and (3.71 and 3.46 individuals) nearby trees. While there was significant difference in the level of abundance of hooded crow in only one habitat nearby field crops with mean
(1.46 and 1.84 individuals) during December 2018 till November 2020. The monthly distribution of hooded crow showed a significant difference in bird population over the study period, data in the Table illustrate that the highest population of hooded crow birds were in September 2019 and 2020 with same mean (4.63 individuals), followed by March, February, April, August, May and October in 2019 with means $(3.88,3.50$, $3.38,3.38,3.25$ and 3.13 individuals) and March, February, April, August and October in 2020 with means $(3.63,3.38$, $3.25,3.00$ and 3.00 individuals), followed by June, July and November 2019 with 
same mean (2.88 individuals) and July, May, June, and November 2020 with means $(2.88,2.75,2.63$ and 2.25 individuals). While the lowest population were in December 2018 and 2019 and Jan. 2019 and 2020 with means (1.63 and 2.00 individuals) and (2.00 and 1.75 individuals). Mosallm (2017) revealed that the lowest values of hooded crow birds were in December 2014 (0.83 individuals) in Beni-Auday city (Manfalot district) at Assiut governorate, Egypt.

Table (2): Population fluctuation and monthly distribution of hooded crow Corvus corone sardonius at different habitats in Assiut governorate, Egypt during December 2018 till November 2020.

\begin{tabular}{|c|c|c|c|c|c|c|c|c|c|c|c|c|c|}
\hline \multirow{3}{*}{ Months } & \multicolumn{12}{|c|}{ Different habitats } & \multirow{3}{*}{ Mean } \\
\hline & \multicolumn{3}{|c|}{ Animal's farm } & \multicolumn{3}{|c|}{ Field crops } & \multicolumn{3}{|c|}{ Honeybee apiary } & \multicolumn{3}{|c|}{ Trees } & \\
\hline & S. R. & S. S. & Av. & S. R. & S. S. & Av. & S. R. & S. S. & Av. & S. R. & S. S. & Av. & \\
\hline December 2018 & 3 & 3 & 3.00 & 1 & 0 & 0.50 & 2 & 1 & 1.50 & 2 & 1 & 1.50 & $1.63 \mathrm{~d}$ \\
\hline January 2019 & 2 & 4 & 3.00 & 2 & 0 & 1.00 & 2 & 2 & 2.00 & 2 & 2 & 2.00 & $2.00 \mathrm{bcd}$ \\
\hline February 2019 & 5 & 3 & 4.00 & 1 & 2 & 1.50 & 3 & 2 & 2.50 & 5 & 7 & 6.00 & $3.50 \mathrm{abcd}$ \\
\hline March 2019 & 6 & 5 & 5.50 & 2 & 2 & 2.00 & 3 & 5 & 4.00 & 2 & 6 & 4.00 & $3.88 \mathrm{ab}$ \\
\hline April 2019 & 5 & 5 & 5.00 & 0 & 3 & 1.50 & 3 & 3 & 3.00 & 3 & 5 & 4.00 & 3.38abcd \\
\hline May 2019 & 4 & 6 & 5.00 & 4 & 1 & 2.50 & 4 & 3 & 3.50 & 2 & 2 & 2.00 & $3.25 \mathrm{abcd}$ \\
\hline June 2019 & 3 & 2 & 2.50 & 0 & 2 & 1.00 & 5 & 4 & 4.50 & 3 & 4 & 3.50 & $2.88 \mathrm{abcd}$ \\
\hline July 2019 & 3 & 2 & 2.50 & 0 & 0 & 0.00 & 4 & 7 & 5.50 & 5 & 2 & 3.50 & $2.88 \mathrm{abcd}$ \\
\hline August 2019 & 5 & 6 & 5.50 & 1 & .0 & 0.50 & 3 & 6 & 4.50 & 3 & 3 & 3.00 & 3.38abcd \\
\hline September 2019 & 4 & 3 & 3.50 & 2 & 4 & 3.00 & 6 & 7 & 6.50 & 4 & 7 & 5.50 & $4.63 a$ \\
\hline October 2019 & 3 & 2 & 2.50 & 3 & 3 & 3.00 & 3 & 2 & 2.50 & 4 & 5 & 4.50 & $3.13 \mathrm{abcd}$ \\
\hline November 2019 & 2 & 3 & 2.50 & 1 & 1 & 1.00 & 4 & 2 & 3.00 & 6 & 4 & 5.00 & 2.88abcd \\
\hline Mean & 3.75 & 3.67 & $3.71 \mathrm{a}$ & 1.42 & 1.50 & $1.46 \mathrm{~b}$ & 3.50 & 3.67 & $3.58 \mathrm{a}$ & 3.42 & 4.00 & $3.71 \mathrm{a}$ & $3.12 \mathrm{a}$ \\
\hline December 2019 & 2 & 3 & 2.50 & 1 & 2 & 1.50 & 1 & 1 & 1.00 & 2 & 4 & 3.00 & $2.00 \mathrm{bcd}$ \\
\hline January 2020 & 3 & 2 & 2.50 & 1 & 2 & 1.50 & 2 & 2 & 2.00 & 1 & 1 & 1.00 & $1.75 \mathrm{~cd}$ \\
\hline February 2020 & 4 & 2 & 3.00 & 3 & 4 & 3.50 & 3 & 2 & 2.50 & 4 & 5 & 4.50 & 3.38abcd \\
\hline March 2020 & 5 & 5 & 5.00 & 2 & 3 & 2.50 & 4 & 4 & 4.00 & 3 & 3 & 3.00 & $3.63 \mathrm{abc}$ \\
\hline April 2020 & 3 & 7 & 5.00 & 2 & 1 & 1.50 & 3 & 6 & 4.50 & 2 & 2 & 2.00 & $3.25 \mathrm{abcd}$ \\
\hline May 2020 & 4 & 4 & 4.00 & 1 & 1 & 1.00 & 4 & 3 & 3.50 & 2 & 3 & 2.50 & $2.75 \mathrm{abcd}$ \\
\hline June 2020 & 4 & 3 & 3.50 & 0 & 1 & 0.50 & 2 & 5 & 3.50 & 4 & 2 & 3.00 & $2.63 \mathrm{bcd}$ \\
\hline July 2020 & 2 & 3 & 2.50 & 1 & 1 & 1.00 & 3 & 5 & 4.00 & 4 & 4 & 4.00 & 2.88abcd \\
\hline August 2020 & 4 & 4 & 4.00 & 2 & 2 & 2.00 & 3 & 2 & 2.50 & 5 & 2 & 3.50 & $3.00 \mathrm{abcd}$ \\
\hline September 2020 & 2 & 3 & 2.50 & 4 & 4 & 4.00 & 5 & 7 & 6.00 & 6 & 6 & 6.00 & $4.63 \mathrm{a}$ \\
\hline October 2020 & 2 & 2 & 2.00 & 2 & 3 & 2.50 & 4 & 2 & 3.00 & 4 & 5 & 4.50 & $3.00 \mathrm{abcd}$ \\
\hline November 2020 & 2 & 2 & 2.00 & 1 & 0 & 0.50 & 2 & 2 & 2.00 & 3 & 6 & 4.50 & $2.25 \mathrm{bcd}$ \\
\hline Mean & 3.08 & 3.33 & $3.21 \mathrm{a}$ & 1.67 & 2.00 & $1.84 \mathrm{~b}$ & 3.00 & 3.42 & $3.21 \mathrm{a}$ & 3.33 & 3.58 & $3.46 \mathrm{a}$ & $2.96 a$ \\
\hline
\end{tabular}

S.R. = sun-rise, S.S. = sun-set, Av. = Average number, *Means have the same litters are not significantly differed by using Duncan's analysis.

\subsubsection{Population fluctuation of palm dove Streptopilia senegalensis egyptica}

Data in Table (3) revealed that the highest population were in fields nearby trees during December 2018 till November 2020 with means $(5.50$ and 5.63 individuals), followed by that recorded in fields nearby honeybee apiary with means
(5.00 and 4.75 individuals), followed by the population in fields nearby animal's farm with means (4.13 and 4.13 individuals). While the lowest numbers were recorded in fields nearby field crops with means (3.38 and 3.29 individuals), during December 2018 till November 2020. Data in the Table (3) showed that the level of abundance of palm dove birds 
that recorded in December 2018 and 2019 with means ( 3.25 and 3.00 individuals). The next months, January 2019 and 2020, recorded the lowest level of abundance of palm dove birds with means $(2.50$ and 2.50 individuals).

Table (3): Population fluctuation and monthly distribution of palm dove Streptopilia senegalensis egyptica at different habitats in Assiut governorate, Egypt during December 2018 till November 2020.

\begin{tabular}{|c|c|c|c|c|c|c|c|c|c|c|c|c|c|}
\hline \multirow{3}{*}{ Months } & \multicolumn{12}{|c|}{ Different habitats } & \multirow{3}{*}{ Mean } \\
\hline & \multicolumn{3}{|c|}{ Animal's farm } & \multicolumn{3}{|c|}{ Field crops } & \multicolumn{3}{|c|}{ Honeybee apiary } & \multicolumn{3}{|c|}{ Trees } & \\
\hline & S. R. & S. S. & Av. & S. R. & S. S. & Av. & S. R. & S. S. & Av. & S. R. & S. S. & Av. & \\
\hline December 2018 & 2 & 2 & 2.00 & 3 & 2 & 2.50 & 2 & 2 & 2.00 & 6 & 7 & 6.50 & $3.25 \mathrm{efg}$ \\
\hline January 2019 & 3 & 2 & 2.50 & 3 & 3 & 3.00 & 2 & 3 & 2.50 & 2 & 2 & 2.00 & $2.50 \mathrm{~g}$ \\
\hline February 2019 & 3 & 4 & 3.50 & 4 & 2 & 3.50 & 5 & 4 & 4.50 & 3 & 7 & 5.00 & 4.00cdefg \\
\hline March 2019 & 5 & 4 & 4.50 & 6 & 6 & 6.00 & 4 & 4 & 4.00 & 6 & 6 & 6.00 & 5.13 bcde \\
\hline April 2019 & 8 & 7 & 7.50 & 5 & 6 & 5.50 & 8 & 7 & 7.50 & 3 & 4 & 3.50 & $6.00 \mathrm{abc}$ \\
\hline May 2019 & 5 & 5 & 5.00 & 3 & 4 & 3.50 & 9 & 12 & 10.50 & 10 & 11 & 10.50 & $7.38 \mathrm{a}$ \\
\hline June 2019 & 4 & 2 & 3.00 & 2 & 2 & 2.00 & 4 & 6 & 5.00 & 4 & 6 & 5.00 & $3.75 \mathrm{defg}$ \\
\hline July 2019 & 4 & 3 & 3.50 & 0 & 2 & 1.00 & 3 & 3 & 3.00 & 3 & 3 & 3.00 & $2.62 \mathrm{fg}$ \\
\hline August 2019 & 7 & 4 & 5.50 & 3 & 2 & 2.50 & 8 & 6 & 7.00 & 6 & 4 & 5.00 & $5.00 \mathrm{bcde}$ \\
\hline September 2019 & 4 & 5 & 4.50 & 6 & 4 & 5.00 & 9 & 7 & 8.00 & 9 & 7 & 8.00 & $6.38 \mathrm{ab}$ \\
\hline October 2019 & 4 & 4 & 4.00 & 4 & 7 & 5.50 & 4 & 4 & 4.00 & 7 & 5 & 6.00 & $4.88 \mathrm{bcde}$ \\
\hline November 2019 & 2 & 6 & 4.00 & 2 & 0 & 1.00 & 2 & 2 & 2.00 & 5 & 6 & 5.50 & $3.12 \mathrm{efg}$ \\
\hline Mean & 4.25 & 4.00 & $4.13 c$ & 3.42 & 3.33 & $3.38 \mathrm{~d}$ & 5.00 & 5.00 & $5.00 \mathrm{~b}$ & 5.33 & 5.67 & $5.50 \mathrm{a}$ & $4.50 \mathrm{a}$ \\
\hline December 2019 & 4 & 2 & 3.00 & 1 & 3 & 2.00 & 3 & 2 & 2.50 & 5 & 4 & 4.50 & $3.00 \mathrm{efg}$ \\
\hline January 2020 & 3 & 2 & 2.50 & 2 & 1 & 1.50 & 4 & 3 & 3.50 & 2 & 3 & 2.50 & $2.50 \mathrm{~g}$ \\
\hline February 2020 & 3 & 5 & 4.00 & 3 & 2 & 2.50 & 3 & 4 & 3.50 & 6 & 5 & 5.50 & $3.88 \mathrm{cdefg}$ \\
\hline March 2020 & 6 & 6 & 6.00 & 5 & 4 & 4.50 & 5 & 3 & 4.00 & 3 & 6 & 4.50 & 4.75bcdef \\
\hline April 2020 & 7 & 9 & 8.00 & 7 & 5 & 6.00 & 7 & 7 & 7.00 & 6 & 6 & 6.00 & $6.75 \mathrm{ab}$ \\
\hline May 2020 & 3 & 6 & 4.50 & 2 & 3 & 2.50 & 10 & 7 & 8.50 & 12 & 10 & 11.00 & $6.63 \mathrm{ab}$ \\
\hline June 2020 & 4 & 4 & 4.00 & 4 & 2 & 3.00 & 5 & 4 & 4.50 & 3 & 4 & 3.50 & $3.75 \mathrm{defg}$ \\
\hline July 2020 & 5 & 3 & 4.00 & 4 & 1 & 2.50 & 4 & 2 & 3.00 & 6 & 4 & 5.00 & $3.62 \mathrm{defg}$ \\
\hline August 2020 & 4 & 6 & 5.00 & 3 & 3 & 3.00 & 7 & 5 & 6.00 & 7 & 5 & 6.00 & $5.00 \mathrm{bcde}$ \\
\hline September 2020 & 3 & 5 & 4.00 & 5 & 3 & 4.00 & 6 & 9 & 7.50 & 7 & 8 & 7.50 & $5.75 \mathrm{abcd}$ \\
\hline October 2020 & 2 & 3 & 2.50 & 6 & 6 & 6.00 & 5 & 4 & 4.50 & 9 & 6 & 7.50 & 5.13 bcde \\
\hline November 2020 & 2 & 2 & 2.00 & 3 & 1 & 2.00 & 2 & 3 & 2.50 & 3 & 5 & 4.00 & $2.62 \mathrm{fg}$ \\
\hline Mean & 3.83 & 4.42 & $4.13 b c$ & 3.75 & 2.83 & $3.29 \mathrm{c}$ & 5.08 & 4.42 & $4.75 \mathrm{ab}$ & 5.75 & 5.50 & $5.63 \mathrm{a}$ & $4.45 \mathrm{a}$ \\
\hline
\end{tabular}

S.R. = sun-rise, S.S. = sun-set, Av. = Average number, *Means have the same litters are not significantly differed by using Duncan's analysis.

February and March had seen increase of the level of abundance of palm dove birds with means (4.00 and 3.88 individuals) and (5.13 and 4.75 individuals). The level of abundance of palm dove birds continued to increase during April and May 2019 and 2020, reaching a peak with means (6.00 and 6.75 individuals) and (7.38 and 6.63 individuals). Two next months, June and July 2019 and 2020, had seen decrease of the level of abundance of palm dove birds with means (3.75 and 3.75 individuals) and (2.63 and 3.63 individuals). Level of abundance of palm dove birds increased in the three next months, August, September and October with means (5.00 and 5.00 individuals), (6.38 and 5.75 individuals) and (4.88 and 5.13 individuals). November recorded low level of abundance of palm dove birds with means (3.13 and 2.63 individuals). The results agree with El-Sawy (2017) who reported that the high numbers of palm dove, on different habitats under study, were observed that the high mean numbers of birds was recorded at trees 
habitat (2.04, 2.38 individuals) followed by buildings (1.13, 1.50 individuals) and water canals (0.92, 0.96 individuals) at (sun-rise and sunset) respectively, while the least numbers were recorded at crops habitat $(0.92,0.75$ individuals) at (sunrise and sunset) respectively at Alexandria governorate, Egypt. El-Danasory et al. (2020) showed that the highest value of palm dove was in fields nearby trees with mean (3.21 birds), followed by in fields nearby building (2.42 birds). The moderate number was recorded in fields nearby water canals (1.38 birds). While the lowest value of palm dove was in fields nearby field crops $(0.75$ birds $)$ at Sohag governorate, Egypt.

\subsubsection{Population fluctuation of rock dove Columba livia schimperi}

Data in table (4) indicated that the mean number of the rock dove birds during December 2018 till November 2020 two successive years in four different habitats (animal's farm, honeybee apiary, field crops and trees) the highest population were in fields nearby animal's farm during December 2018 till November 2020 with mean $(3.00$ and 3.21 individuals respectively), followed by that recorded in fields nearby trees and fields nearby honeybee apiary with means $(2.38$ and 2.63 individuals respectively) and (2.46 and 2.42 individuals respectively).

Table (3): Population fluctuation and monthly distribution of rock dove Columba livia schimperi at different habitats in Assiut governorate, Egypt during December 2018 till November 2020.

\begin{tabular}{|c|c|c|c|c|c|c|c|c|c|c|c|c|c|}
\hline \multirow{3}{*}{ Months } & \multicolumn{12}{|c|}{ Different habitats } & \multirow{3}{*}{ Mean } \\
\hline & \multicolumn{3}{|c|}{ Animal's farm } & \multicolumn{3}{|c|}{ Field crops } & \multicolumn{3}{|c|}{ Honeybee apiary } & \multicolumn{3}{|c|}{ Trees } & \\
\hline & S. R. & S. S. & Av. & S. R. & S. S. & Av. & S. R. & S. S. & Av. & S. R. & S. S. & Av. & \\
\hline December 2018 & 2 & 5 & 3.50 & 0 & 0 & 0.00 & 2 & 2 & 2.00 & 0 & 2 & 1.00 & $1.63 \mathrm{cde}$ \\
\hline January 2019 & 3 & 3 & 3.00 & 1 & 2 & 1.50 & 0 & 0 & 0.00 & 0 & 0 & 0.00 & $1.13 \mathrm{cde}$ \\
\hline February 2019 & 5 & 4 & 4.50 & 2 & 2 & 2.00 & 2 & 0 & 1.00 & 0 & 0 & 0.00 & $1.88 \mathrm{cde}$ \\
\hline March 2019 & 4 & 6 & 5.00 & 2 & 3 & 2.50 & 2 & 3 & 2.50 & 3 & 0 & 1.50 & 2.88 bcde \\
\hline April 2019 & 3 & 3 & 3.00 & 0 & 2 & 1.00 & 3 & 3 & 3.00 & 2 & 2 & 2.00 & $2.25 \mathrm{cde}$ \\
\hline May 2019 & 0 & 2 & 1.00 & 6 & 5 & 5.50 & 7 & 6 & 6.50 & 8 & 8 & 8.00 & $5.25 \mathrm{a}$ \\
\hline June 2019 & 3 & 2 & 2.50 & 0 & 0 & 0.00 & 0 & 0 & 0.00 & 3 & 0 & 1.50 & $1.00 \mathrm{de}$ \\
\hline July 2019 & 1 & 0 & 0.50 & 0 & 0 & 0.00 & 2 & 2 & 2.00 & 0 & 0 & 0.00 & $0.63 \mathrm{e}$ \\
\hline August 2019 & 5 & 2 & 3.50 & 2 & 2 & 2.00 & 6 & 6 & 6.00 & 3 & 4 & 3.50 & $3.75 \mathrm{abc}$ \\
\hline September 2019 & 3 & 3 & 3.00 & 3 & 2 & 2.50 & 4 & 4 & 4.00 & 4 & 6 & 5.00 & 3.63abcd \\
\hline October 2019 & 2 & 4 & 3.00 & 4 & 4 & 4.00 & 3 & 2 & 2.50 & 4 & 4 & 4.00 & 3.38abcd \\
\hline November 2019 & 3 & 4 & 3.50 & 2 & 3 & 2.50 & 0 & 0 & 0.00 & 2 & 2 & 2.00 & $2.00 \mathrm{cde}$ \\
\hline Mean & 2.83 & 3.17 & $3.00 \mathrm{a}$ & 1.83 & 2.25 & $1.96 \mathrm{~b}$ & 2.58 & 2.33 & $2.46 \mathrm{~b}$ & 2.42 & 2.33 & $2.38 \mathrm{~b}$ & $2.45 \mathrm{a}$ \\
\hline December 2019 & 4 & 2 & 3.00 & 2 & 0 & 1.00 & 0 & 0 & 0.00 & 2 & 0 & 1.00 & $1.25 \mathrm{cde}$ \\
\hline January 2020 & 3 & 4 & 3.50 & 2 & 2 & 2.00 & 0 & 0 & 0.00 & 0 & 0 & 0.00 & $1.38 \mathrm{cde}$ \\
\hline February 2020 & 4 & 4 & 4.00 & 0 & 0 & 0.00 & 2 & 2 & 2.00 & 2 & 1 & 1.50 & $1.88 \mathrm{cde}$ \\
\hline March 2020 & 6 & 6 & 6.00 & 3 & 2 & 2.50 & 0 & 3 & 1.50 & 3 & 2 & 2.50 & 3.13abcde \\
\hline April 2020 & 2 & 4 & 3.00 & 2 & 4 & 3.00 & 3 & 4 & 3.50 & 2 & 2 & 2.00 & 2.88 bcde \\
\hline May 2020 & 2 & 2 & 2.00 & 4 & 5 & 4.50 & 6 & 6 & 6.00 & 9 & 10 & 9.50 & $5.50 \mathrm{a}$ \\
\hline June 2020 & 2 & 3 & 2.50 & 0 & 2 & 1.00 & 2 & 0 & 1.00 & 2 & 0 & 1.00 & $1.38 \mathrm{cde}$ \\
\hline July 2020 & 2 & 2 & 2.00 & 0 & 0 & 0.00 & 2 & 2 & 2.00 & 0 & 0 & 0.00 & $1.00 \mathrm{de}$ \\
\hline August 2020 & 4 & 3 & 3.50 & 1 & 3 & 2.00 & 3 & 4 & 3.50 & 2 & 2 & 2.00 & $2.75 \mathrm{bcde}$ \\
\hline September 2020 & 4 & 4 & 4.00 & 3 & 3 & 3.00 & 3 & 2 & 2.50 & 0 & 4 & 2.00 & 2.88 bcde \\
\hline October 2020 & 2 & 4 & 3.00 & 5 & 4 & 4.50 & 5 & 7 & 6.00 & 4 & 8 & 6.00 & $4.88 \mathrm{ab}$ \\
\hline November 2020 & 2 & 2 & 2.00 & 2 & 2 & 2.00 & 2 & 0 & 1.00 & 3 & 5 & 4.00 & $2.25 \mathrm{cde}$ \\
\hline Mean & 3.08 & 3.33 & $3.21 \mathrm{a}$ & 2.00 & 2.25 & $2.13 b$ & 2.33 & 2.50 & $2.42 \mathrm{~b}$ & 2.42 & 2.83 & $2.63 b$ & $2.60 \mathrm{a}$ \\
\hline
\end{tabular}

S.R. = sun-rise, S.S. = sun-set, Av. = Average number, *Means have the same litters are not significantly differed by using Duncan's analysis. 
While the lowest population were in fields nearby field crops with means (1.96 and 2.13 individuals respectively) during December 2018 till November 2020 two successive years. The monthly distribution of rock dove showed a significant difference in bird population over the study period, data in table (4) illustrate that the population fluctuation of rock dove birds in December 2018 and 2019 and January 2019 and 2020 was low with means (1.75 and 1.25 individuals) and (1.13 and 1.38 individuals). The population fluctuation of rock dove increased in three next months, February, March, April 2019 and 2020, with means (1.88 and 1.88 individuals), (2.88 and 3.13 individuals) and (2.25 and 2.88 individuals). As well as the highest population were in May 2019 and 2020 with means (5.25 and 5.50 individuals). Two next months, June and July 2019 and 2020, had seen decrease of the level of abundance of rock dove birds especially in July it reached its lowest level with means (1.00 and 1.38 individuals) and (0.63 and 1.00 individuals). In August, September, October 2019 and 2020 the population returned to increase again with means (3.75 and 2.75 individuals), (3.63 and 2.88 individuals) and (3.38 and 4.88 individuals). November recorded low level of abundance of palm dove birds with means (2.00 and 2.25 individuals).

\section{References}

Attia, M. A. I. (2013), Studies on some wild bird species at Ismailia governorate, Ph.D. Thesis, Faculty of Agriculture, Al-Azhar University, Egypt, pp. 176.

Bonnah, A. A. M. M. (2007), The damage and control of hooded crow on some plantation in Sohag governorate, Ph.D. Thesis, Faculty of Agriculture, Assiut University, Egypt, pp. 116.

El-Sawy, M. F. S. (2017), Biological and ecological studies on harmful birds of agriculture land at Alexandria governorate, M.Sc. Thesis, Faculty of Agriculture, Al-Azhar University, Egypt, pp. 174.

El-Danasory, M. A. M., Ahmed, M. M., Omar, M. M. A. and Hassan, A. A. G. (2020), "Monthly and seasonal fluctuation study of some harmful birds in old lands at Sohag governorate", Egyptian Journal of Plant Protection Research Institute, Vol. 3 No. 1, pp. 326-332.

Hassan, A. A. G. (2018), Ecological and biological studies on some wild bird species at Sohag governorate, M.Sc. Thesis, Faculty of Agriculture, AlAzhar University, Egypt, pp. 152.

Khattab, M. M., Gabr, W. M., Hussien, S. S. and El-Deeb, H. I. (2001), Damage chronology due to house sparrow to wheat varieties during its ripening stages under the ecosystem of newly reclaimed areas at Sharkia governorate, Egyptian Journal of Applied Science, Vol. 16 No. 11, pp. 264-275. 
Khattab, M. M. (2002), Field study on population fluctuation of Hooded crow, Corvus corone saronius (KleinSchmidt), at Sharkia governorate, $2^{\text {nd }}$ International Conference, Plant Protection Research Institute, Cairo, Egypt, pp. 115-120.

Metwally, A. M., El-Deeb, H. H. and Abd El-Aal, S. and Khataab, M. M. (1995), "Birds damage to some ripening field crops under different conditions in Sharkia governorate", Al-Azhar Journal of Agricultural Research, Vol. 21, pp. 413-424.

Metwally, A. M., Ahmed, M. A., Mahmoud, N. A. and Abdel-Aal, M. M. (2009), "Field trials to evaluate damage caused by wild birds to certain field crops under different habitats at Assiut governorate", Mansoura University Journal of Agricultural Sciences, Vol. 34 No. 2, pp. 941-950.

Mosallm, M. A. S. (2017), Ecological and toxicological studies on some wild bird species at Assiut governorate, M.Sc. Thesis Faculty of Agriculture, Al-Azhar University, Egpt, pp. 110.
Omar, M. M. A. (2010), Studies on some wild birds in Assiut with special reference to harmful birds and its control, Ph.D. Thesis Faculty of Agriculture, Al-Azhar University, Egypt, pp. 180.

Omar, M. M. A. (2018), "Population density of house sparrow passer domesticus niloticus (L.) and assessment of damage caused by it's in some wheat cultivars at Assiut region", Minia Journal of Agricultural Research and Development, Vol. 38 No. 3, pp. 487494.

Redinger, R. F. and Libay, S. L. (1979), Perches courted with glue reduce bird damage in rice field plots, Proceeding of Eighth Bird Control Seminar, Bowling Green State University, Bowling Green, Ohio, USA, pp. 201-206. 